\section{Brn-1 and Brn-2 share crucial roles in the production and positioning of mouse neocortical neurons}

\author{
Yoshinobu Sugitani, ${ }^{1}$ Shigeyasu Nakai, ${ }^{1}$ \\ Osamu Minowa, ${ }^{1,2}$ Miyuki Nishi, ${ }^{1}$ \\ Kou-ichi Jishage, ${ }^{1}$ Hitoshi Kawano, ${ }^{3}$ \\ Kensaku Mori, ${ }^{4}$ Masaharu Ogawa, ${ }^{5}$ and \\ Tetsuo Noda ${ }^{1,2,6,7,8}$ \\ ${ }^{1}$ Department of Cell Biology, JFCR-Cancer Institute, Tokyo \\ 170-8455, Japan; ${ }^{2}$ Mouse Functional Genomics Research \\ Group, RIKEN Genomic Sciences Center, Kanagawa \\ 244-0804, Japan; ${ }^{3}$ Department of Developmental Morphology, \\ Tokyo Metropolitan Institute for Neuroscience, Tokyo \\ 183-8526, Japan; ${ }^{4}$ Department of Physiology, Graduate School \\ of Medicine, University of Tokyo, Tokyo 113-0033, Japan; \\ ${ }^{5}$ Laboratory for Cell Culture Development, Brain Science \\ Institute, RIKEN, Saitama 351-0198, Japan; ${ }^{6}$ Department of \\ Molecular Genetics, Tohoku University School of Medicine, \\ Miyagi 980-8575, Japan; ${ }^{7}$ Core Research for Evolutional \\ Science and Technology, Japan Science and Technology \\ Corporation, Saitama 332-0012, Japan
}

Formation of highly organized neocortical structure depends on the production and correct placement of the appropriate number and types of neurons. POU homeodomain proteins Brn-1 and Brn-2 are coexpressed in the developing neocortex, both in the late precursor cells and in the migrating neurons. Here we show that double disruption of both Brn-1 and Brn-2 genes in mice leads to abnormal formation of the neocortex with dramatically reduced production of layer IV-II neurons and defective migration of neurons unable to express mDab1. These data indicate that Brn-1 and Brn-2 share roles in the production and positioning of neocortical neuron development.

Received January 22, 2002; revised version accepted May 23, 2002.

The mature neocortex is organized into six cell layers, each of which contains neurons with similar morphologies, molecular properties, and projection patterns. The development of this neocortical structure depends on a highly ordered pattern of neuronal production and migration. Cortical neurons that comprise each layer are sequentially produced in the ventricular zone of the dorsal telencephalon (Angevine and Sidman 1961; Takahashi et al. 1999). Although the regulatory factors that function in this sequential production of a variety of layer-specific neurons have not been identified in mam-

[Key Words: POU; Brn-1; Brn-2; mDab1; neocortex] ${ }^{8}$ Corresponding author.

E-MAIL tnoda@ims.u-tokyo.ac.jp; FAX 81-35-394-3893.

Article and publication are at http://www.genesdev.org/cgi/doi/10.1101/ gad.978002. mals, in Drosophila the successive production of different types of cells from neuroblasts has been found to require a temporally stereotyped pattern of expression of a set of transcription factors including the Drosophila POU transcription factors Pdm 1 and Pdm2 (Isshiki et al. 2001). In mammals, newly produced neurons leave their birthplace, migrate toward the cortical surface, and form cortical layers in an inside-out pattern with respect to their time of birth (Angevine and Sidman 1961; Rakic 1972). Recent genetic studies have identified large numbers of functional molecules involved in the migration/ positioning of neocortical neurons (for review, see Rice and Curran 1999).

Brn-1 and Brn-2, members of the mammalian class III POU transcription factor family, are prominently expressed in the embryonic brain, including the neocortex (He et al. 1989). Each single mutant, however, shows abnormalities only in limited brain regions. In Brn-2 mutant neonates, neuronal loss was observed only in the hypothalamic supraoptic and paraventricular nuclei, where Brn-1 is not expressed (Nakai et al. 1995; Schonemann et al. 1995). In Brn-1 mutants, remarkable changes in brain morphology were observed only in the hippocampus, where Brn-2 expression is barely detectable (data not shown). In the neocortex, where both Brn-1 and Brn-2 are expressed, no overt developmental defects were seen in either single mutant. These observations suggest functional complementation between Brn-1 and Brn-2 in neocortical development.

\section{Results and Discussion}

To explore their possible overlapping functions in neocortical development, we generated Brn-1/Brn-2 double homozygous mutants by intercrossing double heterozygotes that were healthy and fertile, with no apparent phenotype. Double homozygous mutants were born at the expected Mendelian ratio (76 double homozygous mutants among 1192 pups), but all of them died within $1 \mathrm{~h}$ after birth. In contrast to the limited abnormalities in Brn-1-1- or Brn-2 $2^{-/-}$single mutants, Brn-1/Brn-2 double mutants suffered severe, broad brain defects. The olfactory bulb showed hypoplasia (Fig. 1A,B), and the cerebellum was less foliated, with loosely packed Purkinje cells (Fig. 1C,D). The neocortex was severely affected; its thickness was markedly reduced, and the stratification of the cortical neurons appeared to be disorganized (Fig. 1E,F).

The hypoplastic neocortex could be caused by reduced cell proliferation or accelerated cell death during embryonic corticogenesis. Because there was no evidence of increased apoptosis in Brn-1/Brn-2 double mutant cortex from embryonic day 14.5 (E14.5) to postnatal day 0 (P0; data not shown), we examined the proliferation of cortical progenitor cells by bromodeoxyuridine (BrdU) labeling. In mice, most cortical plate neurons are produced in the ventricular zone (VZ) or in the subventricular zone (SVZ) from E12.5 to E16.5 (The Boulder Committee 1970; Takahashi et al. 1999). Up to E13.5, there was no significant difference in the number of BrdU-labeled cells in the VZ of the double mutant embryos, compared with wild-type (E12.5: $100.0 \% \pm 1.8 \%$ of wild-type; E13.5: $100.8 \% \pm 2.2 \%$ of wild-type; Fig. $\left.2 \mathrm{~A}, \mathrm{~A}^{\prime}\right)$. Reduced 


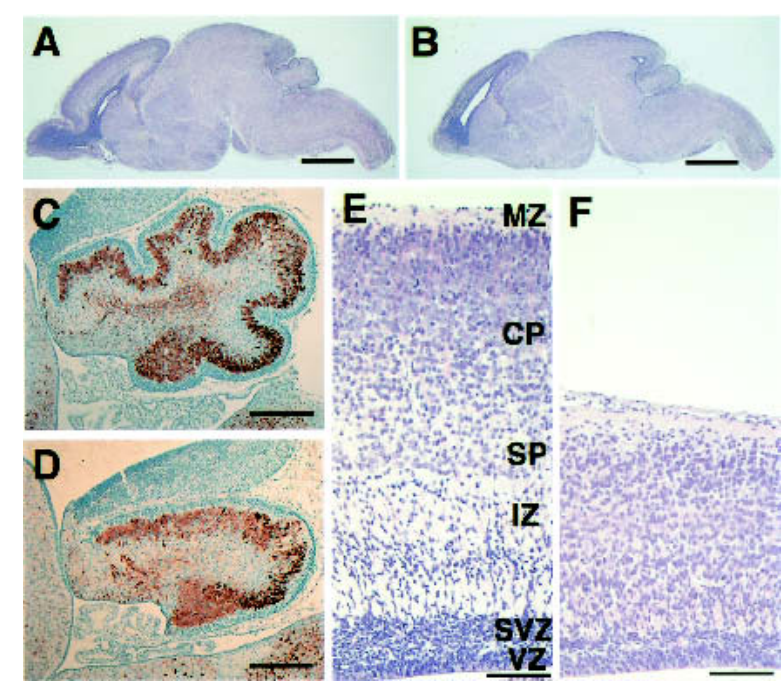

Figure 1. Morphological alterations in Brn-1/Brn-2 double mutant P0 brains. Sagittal sections of whole brain (HE stain) $(A, B)$, cerebellum (brown, anti-Calbindin; blue, hematoxylin) $(C, D)$, and neocortex (HE stain) $(E, F)$ of wild-type $(A, C, E)$ and Brn-1/ Brn-2 double mutant $(B, D, F)$ mice. Normally laminated structure consisting of the ventricular zone (VZ), subventricular zone (SVZ), intermediate zone (IZ), subplate (SP), cortical plate (CP), and marginal zone $(\mathrm{MZ})$ is observed in wild-type neocortex $(E)$, whereas, in Brn-1/Brn-2 mutant neocortex, the overall thickness is markedly reduced and the IZ is not clearly distinguishable from the CP $(F)$. Scale bar: $(A, B) 1 \mathrm{~mm},(C, D) 200 \mu \mathrm{m},(E, F)$ $100 \mu \mathrm{m}$.

cell proliferation in the VZ was observed at E14.5 and thereafter in Brn-1/Brn-2 mutant neocortex. (E14.5: $63.4 \% \pm 2.6 \%$ of wild-type; E16.5: $60.2 \% \pm 3.4 \%$ of wildtype; Fig. $\left.2 \mathrm{~B}, \mathrm{~B}^{\prime}, \mathrm{C}, \mathrm{C}^{\prime}\right)$. Reduction in the number of BrdUlabeled cells was particularly severe in the cortical SVZ in the double mutant (E16.5: $15.1 \% \pm 2.5 \%$ of wild-type; Fig. 2C, $\left.\mathrm{C}^{\prime}\right)$. Despite the hypoplasticity of the Brn-1/Brn-2 deficient cortex, expression of GAD67 and calbindin appeared to be unaffected in the E19.0 neocortex (Fig. 3I,J; data not shown), suggesting intact generation and migration of the cortical interneurons, most of which are derived from the ganglionic eminence (Anderson et al. 1997). These results indicate that Brn-1 and Brn-2 share an essential role in the proliferation of cortical precursor cells within the VZ/SVZ from E14.5 onward, and that the reduction in subsequent cortical cell production could result in the hypoplastic neocortex seen in the double mutant neonate. Analysis of the temporal expression pattern for Brn-1 and Brn-2 proteins in the developing wild-type neocortex revealed that their expression in the $\mathrm{VZ}$ is initiated at $\sim \mathrm{E} 14.5$ and is prominent thereafter in the VZ/SVZ (Fig. 2D-I), with a pattern that corresponds with the period of reduced cell proliferation in the neocortex of double mutant embryos. These results suggest that Brn-1 and Brn-2 may function in the proliferation of late cortical progenitor cells in a cell-autonomous manner.

Lineage analyses and birthdating studies suggest that common cortical precursor cells first produce neurons of layer VI and then layer V (at E11.5-E15.5) and, even later, generate neurons destined for layers IV-II (at E14.5E17.0) by successive cell division (Luskin et al. 1988;
Takahashi et al. 1999|. From the late embryonic neurogenesis stage, glial progenitor cells also proliferate and increase their numbers (Berman et al. 1997), differentiating into astrocytes or oligodendrocytes during a postnatal stage. The finding that Brn-1 and Brn-2 function in cell proliferation, specifically at the late neurogenesis stage, prompted us to examine whether Brn-1 and Brn-2 function in the production of upper-layer neurons and/or in the generation/expansion of glial progenitor cells. We assessed the formation of each cortical layer and the status of gliogenesis in the double mutant cortex at E19.0 or E18.5, using the following markers for different layers and glial progenitors: Tbr-1 for layer VI, subplate and SVZ (Fig. 3A); Wnt7b for layer VI (data not shown; Rubenstein et al. 1999), ER81 for layer V (Fig. 3C); $R O R \beta$ for layer IV (Fig. 3E; Weimann et al. 1999), mSorLA or Svet1 for layers II/III and SVZ cells (Fig. 3G; data not shown; Hermans-Borgmeyer et al. 1998; Tarabykin et al. 2001),
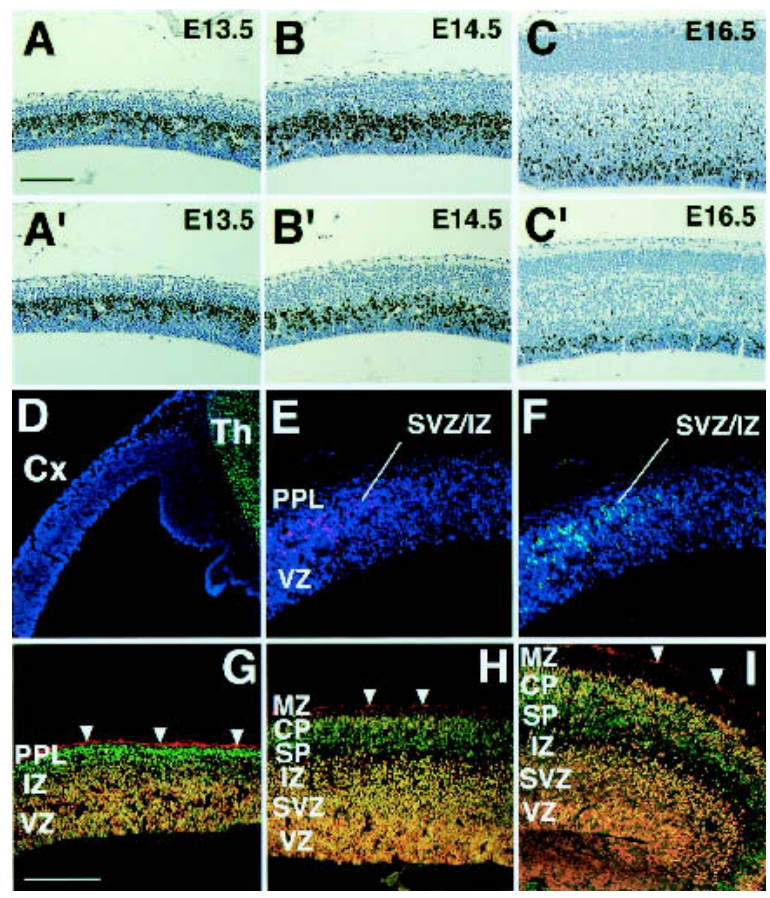

Figure 2. Reduced cell proliferation in Brn-1/Brn-2 mutant neocortex and expression of Brn-1 and Brn-2 in developing neocortex. BrdU labeling (brown) in sagittal sections of wild $(A-C)$ and Brn-1/Brn-2 mutant $\left(A^{\prime}-C^{\prime}\right)$ neocortex at indicated stages. Fluorescent micrographs of sagittal sections of E12.5 (D), E13.5 $(E, F)$, E14.5 $(G)$, E15.5 $(H)$, and E16.5 $(I)$ cortices doubly stained with anti-Brn-1 (red) and anti-Brn-2 (green). Cells expressing both Brn-1 and Brn-2 appear yellow $(G-I)$. All nuclei were stained with DAPI (blue) in $D-F$. Arrowheads indicate background staining by secondary antibodies in pia matter $(G-I)$. From E13.5, Brn-1-expressing and Brn-2-expressing cells are clearly seen outside of the VZ in rostral and lateral cortex $(E, F)$. From E14.5, Brn-1/Brn-2 coexpression also becomes prominent in the VZ/SVZ as well as in the IZ and the CP $(G-I)$. Brn-1 or Brn-2 singularly expressing cells are also found within the $\mathrm{MZ}$ $(H, I)$ or in the PPL $(G)$ and the presumptive SP $(H, I)$, respectively. (Cx) Neocortex, (Th) thalamus, (PPL) preplate. For other abbreviations, see Fig. 1. Scale bar: $\left(A-C, A^{\prime}-C^{\prime}\right) 100 \mu \mathrm{m},(D-I)$ $200 \mu \mathrm{m}$. 


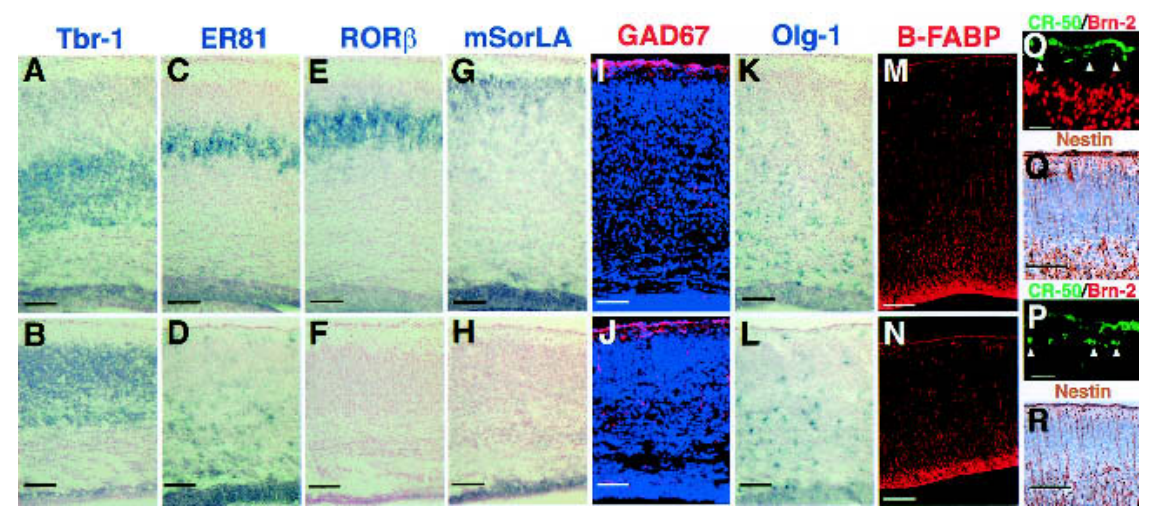

Figure 3. Loss of upper-layer neurons and altered positioning of cortical neurons in Brn-1/Brn-2 mutant neocortex. In situ hybridization using Tbr-1 $(A, B)$, ER81 $(C, D)$, $R O R \beta(E, F), \operatorname{mSorLA}(G, H), \operatorname{Olg}-1(K, L)$ riboprobes on coronal sections of E19.0 wild-type $(A, C, E, G, K)$ and Brn-1/Brn-2 mutant $(B, D, F, H, L)$ cortices. In wild-type cortex, Tbr-1positive layer VI, ER81-positive layer V, ROR $\beta$-positive layer IV, and $m S o r L A$-positive layer II/III neurons are ordered from deep to superficial $(A, C, E, G)$. In Brn-1/Brn-2 mutant cortex, however, the majority of the ER81-positive neurons are found beneath the Tbr1-positive layer, with a few ER81-positive neurons detected in the superficial region within the cortical plate $(B, D)$, and the numbers of layer IV or layer II/III neurons positive for $R O R \beta$ or $m$ Sor $L A$ are drastically reduced $(F, H)$, although $m$ Sor $L A$ expression is found in the SVZ with a similar pattern of Tbr-1 expression in the SVZ $(H, B)$. Immunostaining against GAD67 (red) $(I, J)$, B-FABP (red) $(M, N)$, Reelin (green) and Brn-2 (red) $(O, P)$, and Nestin (brown) $(Q, R)$ on sagittal $(M, N, Q, R)$ and coronal $(I, J, O, P)$ sections of E19.0 $(I, J)$, E18.5 $(M-P)$ and E16.5 $(Q, R)$ cortices of wild-type $(I, M, O, Q)$ and Brn-1/Brn-2 mutant $(J, N, P, R)$. Scale bar: $(A-N) 100 \mu \mathrm{m},(O, P) 20 \mu \mathrm{m},(Q, R) 50 \mu \mathrm{m}$.

Olg-1 for oligodendrocyte progenitors (Fig. 3K; Lu et al. 2000; Zhou et al. 2000), B-FABP/BLBP for immature astrocytes and radial glial cells (Fig. 3M; Feng et al. 1994; Kurtz et al. 1994), and CR-50 for Cajal-Retzius neurons in the marginal zone (MZ; Fig. 3O; Ogawa et al. 1995; D'Arcangelo et al. 1997). The marker studies indicated that the initial step of gliogenesis seemed to be unaffected in Brn-1/Brn-2 mutant neocortex (Fig. 3L,N), whereas the numbers of RORß-positive, mSorLA-positive, or Svet1-positive neurons were dramatically reduced in Brn-1/Brn-2 mutant neocortex with mSorLAexpressing or Svet1-expressing SVZ cells lining the entire surface of the enlarged lateral ventricles of the mutant brains (Fig. 3F, H; data not shown). These results suggest that Brn-1 and Brn-2 are essential for proper production of neocortical neurons destined for layers VI-II.

Molecular marker analysis also revealed abnormal layering of the remaining cortical neurons in Brn-1/Brn-2deficient neocortex, in which the majority of ER81-positive layer V neurons, normally laminated above the $\mathrm{Tbr}$ 1-positive or $W n t 7 b$-positive layer VI (Fig. 3A,C; data not shown), were found beneath the Tbr-1-positive or Wnt $7 b$-positive layer (Fig. 3B,D; data not shown). It has been well documented that the laminar structure of the neocortex is built by migration of successively produced neurons in an inside-to-outside fashion, such that neurons born earlier reside in deeper layers, and those born later occupy more superficial layers within the cortical plate (CP) between the MZ and the subplate (SP). Thus, the largely inverted packing pattern of layer $\mathrm{V}$ and VI neurons in Brn-1/Brn-2 mutant cortex can be caused by either abnormal cell migration or cell fate defects such that the timing of layer VI and layer V neuronogenesis is inverted. To distinguish between the two possibilities, we labeled E12.5, E13.5, and E14.5 embryos, stages during which layer VI-V neuronogenesis is at a peak, with BrdU and examined the localization of BrdU-positive cortical neurons in E19.0 embryos. If the abnormal lamination is caused by cell fate defects, BrdU-labeled neurons should appear in comparable positions in the wild-type and Brn-1/Brn-2 mutant cortices. Conversely, if neuronal migration is affected, neurons labeled at the same time should occupy different positions in wild-type and mutant mice. In E19.0 wild-type cortex, cells born on E12.5 occupied the SP and the deepest part of layer VI (Fig. 4A), and most of the cells at E13.5 predominantly occupied layer VI above the E12.5-born cohort (Fig. 4B). The relative positions of E13.5-born to E12.5born neurons in the Brn-1/Brn-2-deficient cortex at E19.0 (Fig. 4D,E) were comparable with those in their wildtype littermates (Fig. 4A,B). The positioning of E14.5-born neurons, however, was significantly altered. E14.5born cells in wild-type cortex occupied layers $\mathrm{V}$ and IV in a superficial region of the CP (Fig. 4C), whereas those in Brn-1/Brn-2-deficient cortex remained in the intermediate zone (IZ), beneath the cohort of E12.5-born cells (Fig. 4F). Together with the abnormal localization of the layer V neurons in the IZ of Brn-1/Brn-2 mutant cortex (Fig. 3D), these BrdU neural birthdating experiments suggest abnormal migration of the layer $\mathrm{V}$ neurons born after E13.5 in Brn-1/Brn-2 mutant cortex (Fig. $4 \mathrm{~K}^{\prime}, \mathrm{L}^{\prime} \mathrm{L}^{\prime}$ ).

Correct neuronal migration requires both radial glial fibers as guiding scaffolds for migrating neurons (Rakic 1972) and Cajal-Retzius neurons that play a key role in neuronal lamination by producing the secreted Reelin protein (Ogawa et al. 1995; Rice and Curran 1999). The alignment and density of radial glial fibers, labeled with antibodies against B-FABP or Nestin, were not altered (Fig. 3N,R). Furthermore, neither the number of CajalRetzius neurons nor their immunolabeling intensity for Reelin was changed in the Brn-1/Brn-2-deficient cortex (Fig. 3P). In fact, Cajal-Retzius neurons in the wild-type cortex expressed neither Brn-1 nor Brn-2 at E16.5 and E18.5 cortex (Fig. 3O; data not shown). Thus, the migration defects in the Brn-1/Brn-2-deficient cortex do not seem to be a consequence of a disrupted radial glial fiber system or a loss of Reelin-expressing Cajal-Retzius neurons. Given Brn-1/Brn-2 coexpression in migrating neurons both in the IZ and CP (Fig. 2E-I), the altered migration of Brn-1/Brn-2-deficient cortical neurons can be a result of cell-autonomous defects.

To investigate the molecular mechanisms underlying the neuronal migration defects in Brn-1/Brn-2 mutant cortex, an RT-PCR analysis was performed on various genes involved in neuronal migration (Rice and Curran 1999). mDab1, VLDLR/ApoER2, and $\alpha 3$-integrin have been shown to function in positioning cortical neurons by mediating Reelin signal transduction. CDK5, p35 (one of the CDK5 activator subunits), Lis1 (Pafahib1), and 


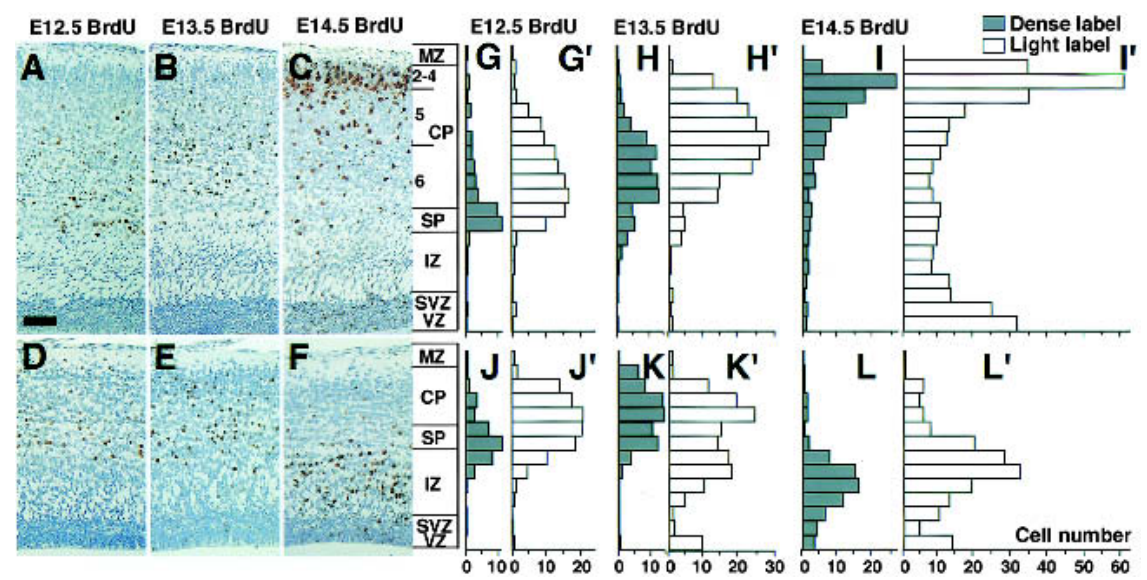

Figure 4. Abnormal migration of Brn-1/Brn-2 mutant neurons. (Left panels) Distribution of cells labeled with BrdU during E12.5 $(A, D)$, E13.5 $(B, E)$, or E14.5 $(C, F)$ in E19.0 wild-type $(A-C)$ and Brn-1/Brn-2 mutant $(D-F)$ sagittal cortical sections. BrdU-positive nuclei (brown) were detected by immunohistochemistry. (Right panels) Bar graphs showing the radial distribution of heavily labeled cells (first generation at time of BrdU injection) $(G-L)$ and lightly labeled cells (the majority of them are second and perhaps third generation cells from subsequent progenitor cell divisions) $\left(G^{\prime}-L^{\prime}\right)$ in E19.0 wildtype $\left(G-I, G^{\prime}-I^{\prime}\right)$ and Brn-1/Brn-2 mutant $\left(J-L, J^{\prime}-L^{\prime}\right)$ neocortex. In Brn-1/Brn-2 mutants, most E14.5 BrdU-labeled cells $\left(L, L^{\prime}\right)$ occupy the deepest positions. The subpopulation of E13.5 lightly labeled cells is also shifted to deeper positions $\left(K^{\prime}\right)$. (2-4) Layer II-VI, (5) Layer V, (6) Layer VI. For other abbreviations, see Fig. 1. Scale bar: $80 \mu \mathrm{m}$.

Doublecortin are also thought to affect neuronal migration in the developing cortex. Among all these tested genes, only mdab1 expression was clearly affected in the Brn-1/Brn-2 double mutant cortex at E16.5 (Fig. 5A,B; data not shown). Therefore, we examined the spatial distribution of the mdab1 mRNA in the cortex of Brn-1/ Brn-2 mutant embryos and wild-type littermates by RNA in situ hybridization. In the wild-type cortex at E16.5, mdab1 mRNA was expressed throughout the cortical wall, except for the MZ and SP. High levels of mdab1 mRNA were detected in the upper regions of the IZ and in the CP (Fig. 5E; Rice and Curran 1999). In the Brn-1/Brn-2-deficient cortex at E16.5, mdab1 mRNA expression was significantly reduced throughout the cortical wall and, in particular, was undetectable in the upper region of the IZ (Fig. 5F) just beneath the chondroitin sulfate proteoglycans (CSPG)-positive SP (Sheppard et al. 1991), in which p35-highly expressing late-born neurons were abnormally congested (Fig. $5 \mathrm{H}, \mathrm{J}, \mathrm{L})$. Therefore, the slight reduction in p35 mRNA levels in the E16.5 mutant cortex detected by RT-PCR analysis (Fig. 5A,B) might be caused by decreased numbers of $p 35$-expressing neurons produced from E14.5 onward. Furthermore, quantitative RT-PCR analysis showed that mdab1 expression was reduced also in Brn-1/Brn-2 double heterozygotes (Fig. 5A,B), which show no histological defects in their neocortex. RNA in situ hybridization also showed that precipitously graded reduction of mdab1 mRNA levels correlated well with Brn-1/Brn-2 gene dosages (data not shown). These results imply that Brn-1 and Brn-2 act genetically upstream to activate mDab1-dependent positioning processes in cortical neurons. The early-born neurons lacking Brn-1 and Brn-2, however, migrate and split the preplate into the MZ and SP properly (Fig. 5J), which is not seen in the mdab1 mutant cortex; in yotari and scrambler, mutant mice carrying loss-of-function mutations in the mdab1 gene, cortical neurons fail to split the preplate to form the CP between the MZ and SP (Rice and Curran 1999). The maintenance of integrity of preplate splitting in Brn-1/ Brn-2 mutant E16.5 cortex could be caused by the redundant function of another class III POU factor, Brn-4, that also shares high homology in its primary structure with Brn-1 and Brn-2 (Mathis et al. 1992). In wildtype as well as double-mutant cortex, Brn-4 expression was also detected in the migrating neurons at $\sim \mathrm{E} 15.5$, but was reduced after then (Fig. 5M-P). In Brn-1/Brn-2 mutant cortex, mDab1 expression was detected until E15.5 (Fig. 5D) but was hardly detectable at E16.5 (Fig. 5F). Therefore, Brn-4, like Brn-1 and Brn-2, might also be able to activate mDab1-dependent processes in the positioning of early-born neurons.

Here we showed that there are two distinct types of the expression pattern of Brn-1/Brn-2 proteins in developing neocortex. Brn-1/Brn-2 expression in the precursor cells is restricted to a late pool of neural precursors, and Brn-1/Brn-2 is also expressed in a wide range of the postmitotic neurons, including Tbr-1-positive cortical plate neurons (data not shown). Double disruption of both Brn-1 and Brn-2 genes in mice led to two types of abnormalities during the neocortical development: selective loss of the neurons positive for layer IV-II markers (ROR $\beta, m$ SorLA, and Svet1), and significantly reduced $m D a b 1$ expression in all remaining neurons at late phase, independently of Brn-1/Brn-2 expression in their precursors.

Several lines of evidence suggest that mDabl functions downstream of Reelin in a signaling pathway that controls cell positioning in the developing cortex (Rice and Curran 1999). However, it is not yet clear how these molecules dictate the spatial position of cortical neurons, including subplate neurons. Interestingly, in the Brn-1/Brn-2-deficient cortex, mDab1 expression was severely reduced only at a late stage, when most of the E14.5-born neurons migrate through the IZ, but do not reach the MZ, remaining congested just beneath the SP. Therefore, these results imply that mDab1 may be necessary for CP neurons to migrate through the SP. Alternatively, Brn-1 and Brn-2 could also regulate expression of other molecules that may be essential in this process. On the other hand, the hypoplasticity of the Brn-1/Brn2-deficient cortex cannot be explained by an inability to express mdab1, because reduced cell proliferation has not been reported in mdab1 mutant cortex, and loss of $R O R \beta$-expressing or $m S o r L A$-expressing neurons was not observed in yotari (data not shown). We examined tailless (Monaghan et al. 1997) and pax6 (Tarabykin et al. 2001) expression, which are known to be essential for proper generation of cortical neurons. However, we found no changes in their expression in Brn-1/Brn-2 mutant cortex (data not shown).

Previous reports have indicated that the earliest events 


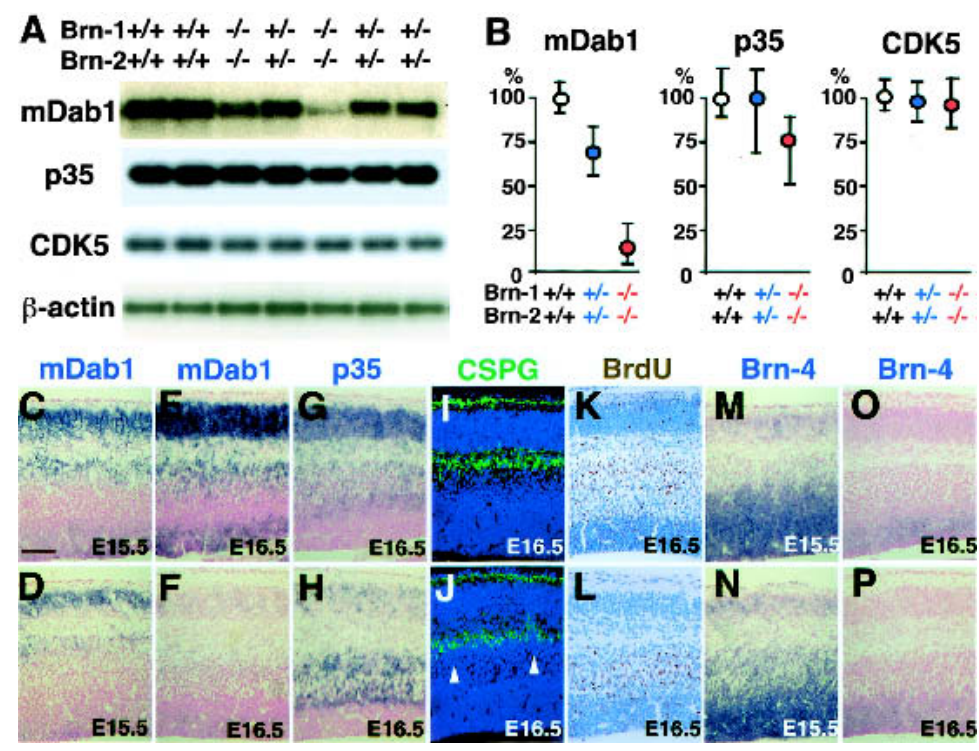

Figure 5. Reduced mDab1 expression level and congestion of migrating neurons just beneath the subplate in Brn-1/Brn-2 mutant cortex. (A) RTPCR analysis for $m D a b 1, p 35, C D K 5$, and $\beta$-actin mRNA expression in the E16.5 dorsal cortex of wild-type $(+/+)$, Brn-1/Brn-2 double heterozygotes $(+/-)$, and Brn-1/Brn-2 double homozygotes $(-/-)$, and $(B)$ quantitation of their mRNA levels in Brn-1/Brn-2 double heterozygotes $(n=5)$ and Brn-1/ Brn-2 double homozygotes $(n=5)$ relative to those of wild-type embryos $(n=4)$. In situ hybridization using $m D a b 1(C-F), p 35(G, H)$, and Brn-4 $(M-P)$ riboprobes (blue) and immunostaining against CSPGs (green) $(I, I)$ and BrdU labeled at E14.5 (brown) $(K, L)$ on sagittal sections of E15.5 $(C, D, M, N)$ and E16.5 $(E-L, O, P)$ wild-type $(C, E, G, I, K, M, O)$ and Brn-1/Brn-2 mutant $(D, F$, $H, J, L, N, P)$ cortices. All nuclei were stained with DAPI (blue) in $I$ and $J$. Although $m D a b 1$ expression is detected in wild-type and Brn-1/Brn-2 mutant cortices $(C, D)$ at E15.5 when Brn-4 is expressed $(M, N)$, it is hardly detectable in E16.5 mutant cortex $(F)$ when and where Brn-4 expression is decreased $(P)$. The $p 35$-expressing neurons in Brn-1/Brn-2 mutant cortex are less abundant in the CP and more so in the IZ beneath the SP $(H)$ compared with wild-type $(G)$. Immunolabeling for CSPGs, which is intense in the MZ and the SP $(I, J)$, shows proper splitting of the preplate into the MZ and SP and abnormal cell congestion just beneath the CSPG-positive SP (white arrowheads) in Brn-1/Brn-2 mutant cortex $(J)$. In the wild-type cortex, the majority of E14.5-born neurons are found in the IZ, and some of them have already entered into the CP $(K)$, whereas in Brn-1/Brn-2 mutant cortex, none of the E14.5-born cells are found in the $\mathrm{CP}$, and all of them stay beneath the presumptive SP $(L)$. Scale bar: $(C-P) 100 \mu \mathrm{m}$.

of cell class specification within each cortical layer occur in coordination with neuronogenesis within the proliferating zone (McConnell and Kaznowski 1991). At later stages, when superficial layers are being generated, the progenitors become restricted to an upper-layer fate (Frantz and McConnell 1996). A recent report suggests that the subpopulation of the SVZ cells derived from the VZ represents neuronal progenitors committed to upperlayer neurons (Tarabykin et al. 2001). Because Brn-1 and Brn-2 are specifically expressed in late precursor cells within the cortical VZ/SVZ and function in the proliferation of these cells both in the VZ and especially in the SVZ, these factors might share an intrinsic role in the production of fate-committed neuronal precursors and/ or cortical neurons destined for the upper layers. Further analysis on these overlapping mutants would provide insight into the developmental mechanisms of the mammalian neocortex with its great diversity of cortical neurons.

\begin{abstract}
Materials and methods
Histology and immunohistochemistry for calbindin, BrdU, and Nestin

Fixed samples in Bouin's fixative were dehydrated and embedded in paraffin blocks, from which 5-8- $\mu \mathrm{m}$ serial sections were cut. Hematoxylin and eosin (HE) staining was performed following standard protocols. For immunohistochemistry, the following antibodies were used: anti-Calbindin (a gift of M. Watanabe, Hokkaido University, Japan), anti-BrdU (Beckton Dickinson), anti-Nestin (a gift of Y. Tomooka, Science University of Tokyo, Japan), and anti-Pax6 (a gift of N. Osumi, Tohoku University, Japan). The Vectastain ABC kit (Vector Laboratories) was used for detection. The sections were counterstained with hematoxylin.
\end{abstract}

\section{BrdU-labeling analysis}

For the cell proliferation assay, we injected pregnant mice intraperitoneally with $\mathrm{BrdU}(50 \mathrm{mg} / \mathrm{kg}) 1.0 \mathrm{~h}$ before death. BrdUpositive cells were visualized as described above. Three embryos for each genotype were analyzed at the indicated stages, and 10 sagittal sections at the level of the olfactory bulb for each embryo were used. The fraction of BrdU-positive cells in the VZ was determined by dividing the number of BrdU-positive nuclei by the total number of the nuclei identified in units of the $200-\mu \mathrm{m}$-wide VZ. For the assay in the SVZ, because of the difficulty in distinguishing SVZ cells from postmitotic cells, BrdU-positive SVZ cells were counted in the same units as the assay in the VZ. For birthdating analysis to determine the distributions of the cells labeled with BrdU $(30 \mathrm{mg} / \mathrm{kg})$ in the E19.0 neocortical wall, parasagittal sections at the level of the accessory olfactory bulb were used. At the level, 500- $\mu$ m-wide radial stripes in the medial portions were divided into $\sim 40-\mu \mathrm{m}$-deep bins $(20$ horizontal bins in wild-type cortex and 14 bins in mutant cortex, respectively), and the position of each heavily and lightly labeled cell was assigned to a bin to generate histograms of the number of labeled cells against depth. Data from five sections from each of two to three littermates were averaged to give the histograms.

\section{Immunofluorescence, apoptosis assay, and RNA in} situ hybridization

Fixed samples with $4 \%$ paraformaldehyde in PBS were embedded in OCT compound, and serial sections $(6-30 \mu \mathrm{m})$ were cut using a cryostat and immunostained with the following primary antibodies: anti-GAD (Chemicon, 1:1500 dilution), antiB-FABP (a gift of F. Spener, University of Münster, Germany), CR-50 (1:100 dilution), anti-Brn-1 (Santa Cruz, 1:80 dilution), anti-CSPGs (Sigma, 1:600 dilution), and anti-Brn-2 (1:800 dilution). Anti-Brn-2 rabbit polyclonal antibodies were raised against the $C$ terminus of Brn-2 (amino acids 422-433). Western blot analysis and immunostaining confirmed the specificity of the antibodies. Apoptosis in the cortex at E14.5-P0 was assayed by using a TUNEL assay kit (Oncor). RNA in situ hybridization was performed by modified protocols as described earlier (Minowa et al. 1999). Riboprobes were synthesized using the following murine cDNAs: mdab1 (30-464), p35 (1142-1791), Brn-4 (2219-2600), Tbr-1 (2207-2956), Wnt7b (11381449), mSorLA (5320-6101), Svet1 (2622-3243), and tailless (729-1410).

\section{RT-PCR analysis}

Total RNA $(7.5 \mu \mathrm{g})$, extracted from dissected E16.5 dorsal cortices of each embryo, were reverse-transcribed with an oligo(dT) primer (Invitrogen), and $1 / 20$ of each RT reaction was subjected to PCR amplification using specific primer pairs. The $\beta$-actin gene was used as control. The primer pairs used were mDab1, 5'-GGGCTGGAGAGCGCGTTTGAGTGCG3', 5'CTTCATCATGGAATCTTGACATAAC-3'; p35, 5'-TCGGCTGC TGACCACTCACTTTCCG-3', 5'-AACAAAGATCACGGGCACCAGC GAG-3'; CDK5 , 5' -CTAATGCAGGACGACCTCTCTTCCC-3' ' 5'-TCA GCATCCCACACCCGACTCTTCC- 3 '; and $\beta$-actin $\left(5^{\prime}\right.$-CCTTCAACAC CCCAGCCATG-3', 5'-TGCGCTCAGGAGGAGCAATG-3'. The PCR 
products obtained were subjected to electrophoresis, and the intensities of each amplified band were analyzed by densitometry. The PCR products for mDab1, p35, CDK5, and $\beta$-actin were transferred to nylon-based membranes and hybridized with the following ${ }^{32} \mathrm{P}$-labeled oligonucleotides specific for each cDNA: mDab1, 5'AAGGTCAGGATCGCAGC GAAGCCAC-3'; p35, 5'-TCCCCACTGTCCCATGATCGGAGCTG-3'; CDK5, 5'-CCCCATAGGCTCTCTGAACCCCAGT-3'; and $\beta$-actin, $5^{\prime}$ CAAGTCATCACTATTGGCAACGA-3'. For relative quantitation of $m D a b 1, p 35$, and CDK5 mRNA, the radioactivity of the amplified bands was quantitated relative to standard curves obtained by PCR amplification of serially diluted wild-type RT-products.

\section{Acknowledgments}

We thank M. Watanabe, Y. Tomooka, F. Spener, and N. Osumi for their gifts of rabbit polyclonal antibodies; and K. Okubo, T.M. Jessell, S.K McConnell, and D.H. Rowritch for their gifts of the doublecortin, the ER81, the ROR $\beta$, and the Olg-1 probes.

The publication costs of this article were defrayed in part by payment of page charges. This article must therefore be hereby marked "advertisement" in accordance with 18 USC section 1734 solely to indicate this fact.

\section{References}

Anderson, S.A., Eisenstat, D.D., Shi, L., and Rubenstein, J.L. 1997. Interneuron migration from basal forebrain to neocortex: Dependence on Dlx genes. Science 278: 474-476.

Angevine, J.B. and Sidman, R.L. 1961. Autoradiographic study of cell migration during histogenesis of the cerebral cortex in the mouse. Nature 192: 766-768.

Berman, N.E., Johnson, J.K., and Klein, R.M. 1997. Early generation of glia in the intermediate zone of the developing cerebral cortex. Brain Res. Dev. Brain Res. 101: 149-164.

The Boulder Committee. 1970. Embryonic vertebrate central nervous system: Revised terminology. Anat. Rec. 166: 257-261.

D’Arcangelo, G., Nakajima, K., Miyata, T., Ogawa, M., Mikoshiba, K. and Curran, T. 1997. Reelin is a secreted glycoprotein recognized by the CR-50 monoclonal antibody. J. Neurosci. 17: 23-31.

Feng, L., Hatten, M.E., and Heintz, N. 1994. Brain lipid-binding protein (BLBP): A novel signaling system in the developing mammalian CNS. Neuron 12: 895-908.

Frantz, G.D. and McConnell, S.K. 1996. Restriction of late cerebral cortical progenitors to an upper-layer fate. Neuron 17: 55-61.

He X., Treacy, M.N., Simmons, D.M, Ingraham, H.A., Swanson, L.W., and Rosenfeld, M.G. 1989. Expression of a large family of POU-domain regulatory genes in mammalian brain development. Nature 340: 35-41.

Hermans-Borgmeyer, I., Hampe, W., Schinke, B., Methner, A., Nykjaer, A., Susens, U., Fenger, U., Herbarth, B., and Schaller, H.C. 1998. Unique expression pattern of a novel mosaic receptor in the developing cerebral cortex. Mech. Dev. 70: 65-76.

Isshiki, T., Pearson, B., Holbrook, S., and Doe, C.Q. 2001. Drosophila neuroblasts sequentially express transcription factors which specify the temporal identity of their neuronal progeny. Cell 106: 511-521.

Kurtz, A., Zimmer, A., Schnutgen, F., Bruning, G., Spener, F., and Muller, T. 1994. The expression pattern of a novel gene encoding brain-fatty acid binding protein correlates with neuronal and glial cell development. Development 120: 2637-2649.

Lu, Q.R., Yuk, D., Alberta, J.A., Zhu, Z., Pawlitzky, I., Chan, J., McMahon, A.P., Stiles, C.D., and Rowitch, D.H. 2000. Sonic hedgehog: Regulated oligodendrocyte lineage genes encoding bHLH proteins in the mammalian central nervous system. Neuron 25: 317-329.

Luskin, M.B., Pearlman, A.L., and Sanes, J.R. 1988. Cell lineage in the cerebral cortex of the mouse studied in vivo and in vitro with a recombinant retrovirus. Neuron 8: 635-647.

Mathis, J.M., Simmons, D.M., He, X., Swanson, L.W., and Rosenfeld, M.G. 1992. Brain 4: A novel mammalian POU domain transcription factor exhibiting restricted brain-specific expression. EMBO J. 11: 2551-2561.

McConnell, S.K. and Kaznowski, C.E. 1991. Cell cycle dependence of laminar determination in developing neocortex. Science 254: 282285 .
Minowa, O., Ikeda, K., Sugitani, Y., Oshima, T., Nakai, S., Katori, Y., Suzuki, M., Furukawa, M., Kawase, T., Zheng, Y., et al. 1999. Altered cochlear fibrocytes in a mouse model of DFN3 nonsyndromic deafness. Science 285: 1408-1411.

Monaghan, A.P., Bock, D., Gass, P., Schwager, A., Wolfer, D.P., Lipp, H.P., and Schutz, G. 1997. Defective limbic system in mice lacking the tailless gene. Nature 390: 515-517.

Nakai, S., Kawano, H., Yudate, T., Nishi, M., Kuno, J., Nagata, A., Jishage, K., Hamada, H., Fujii, H., Kawamura, K., et al. 1995. The POU domain transcription factor Brn-2 is required for the determination of specific neuronal lineages in the hypothalamus of the mouse. Genes \& Dev. 9: 3109-3121.

Ogawa, M., Miyata, T., Nakajima, K., Yagyu, K., Seike, M., Ikenaka, K., Yamamoto, H., and Mikoshiba, K. 1995. The reeler gene-associated antigen on Cajal-Retzius neurons is a crucial molecule for laminar organization of cortical neurons. Neuron 14: 899-912.

Rakic, P. 1972. Mode of cell migration to the superficial layers of fetal monkey neocortex. J. Comp. Neurol. 145: 61-83.

Rice, D.S. and Curran, T. 1999. Mutant mice with scrambled brains: Understanding the signaling pathways that control cell positioning in the CNS. Genes \& Dev. 13: 2758-2773.

Rubenstein, J.L., Anderson, S., Shi, L., Miyashita-Lin, E., Bulfone, A., and Hevner, R. 1999. Genetic control of cortical regionalization and connectivity. Cereb. Cortex 9: 524-532.

Schonemann, M.D., Ryan, A.K., McEvilly, R.J., O'Connell, S.M., Arias, C.A., Kalla, K.A., Li, P., Sawchenko, P.E., and Rosenfeld, M.G. 1995 Development and survival of the endocrine hypothalamus and posterior pituitary gland requires the neuronal POU domain factor Brn-2. Genes \& Dev. 9: 3122-3135.

Sheppard, A.M., Hamilton, S.K., and Pearlman, A.L. 1991. Changes in the distribution of extracellular matrix components accompany early morphogenetic events of mammalian cortical development. I. Neurosci. 11: 3928-3942.

Takahashi, T., Goto, T., Miyama, S., Nowakowski, R.S., and Caviness, V.S., Jr. 1999. Sequence of neuron origin and neocortical laminar fate: Relation to cell cycle of origin in the developing murine cerebral wall. J. Neurosci. 19: 10357-10371.

Tarabykin, V., Stoykova, A., Usma, N., and Gruss, P. 2001. Cortical upper layer neurons derive from the subventricular zone as indicated by Svet1 gene expression. Development 128: 1983-1993.

Weimann, J.M., Zhang, Y.A., Levin, M.E., Devine, W.P., Brulet, P., and McConnell, S.K. 1999. Cortical neurons require Otxl for the refinement of exuberant axonal projections to subcortical targets. Neuron 24: 819-831.

Zhou, Q., Wang, S., and Anderson, D.J. 2000. Identification of a novel family of oligodendrocyte lineage-specific basic helix-loop-helix transcription factors. Neuron 25: 331-343. 


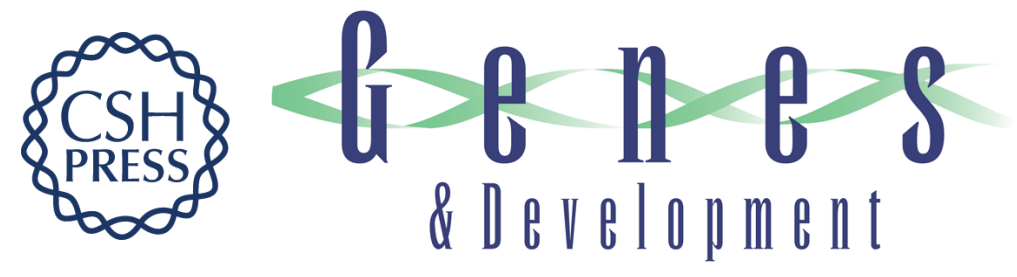

\section{Brn-1 and Brn-2 share crucial roles in the production and positioning of mouse neocortical neurons}

Yoshinobu Sugitani, Shigeyasu Nakai, Osamu Minowa, et al.

Genes Dev. 2002, 16:

Access the most recent version at doi:10.1101/gad.978002

\section{Supplemental http://genesdev.cshlp.org/content/suppl/2002/07/30/16.14.1760.DC1 Material}

References This article cites 27 articles, 11 of which can be accessed free at: http://genesdev.cshlp.org/content/16/14/1760.full.html\#ref-list-1

\section{License}

Email Alerting

Receive free email alerts when new articles cite this article - sign up in the box at the top Service 